\title{
Contribution à la connaissance du fonctionnement des grands fleuves Structure et dynamique des peuplements macrobenthiques de la Loire Analyse des facteurs de micro-distribution
}

\section{Contribution to the knowledge of the functioning of large rivers. Structure and dynamics of macrobenthic populations of the Loire river Analysis of micro distribution factors}

\author{
Michel Bacchi \\ Professeur Associé de l'Université de Tours - RIVE Sarl - 5, rue Carnot - 37500 Chinon \\ E.mail : rive.chinon@wanadoo.fr
}

\section{Jean-Pierre Berton}

\begin{abstract}
Professeur de l'Université de Tours - IMACOF - Faculté des Sciences de Tours Parc de Grandmont - 37200 Tours

E.mail : jpberton@univ-tours.fr
\end{abstract}

Analyse des facteurs de micro-distribution des invertébrés benthiques en Loire

Résumé. - La détermination, dans un complexe de facteurs, des principaux paramètres influant sur les biocénoses est une nécessité pour comprendre le fonctionnement d'un hydrosystème et donc pour en assurer la meilleure gestion. Ce travail, mené dans le cadre du Plan Loire Grandeur Nature, présente la comparaison et la hiérarchisation des principaux facteurs de micro-distribution observés sur deux stations ligériennes : Bréhémont et La Charité-sur-Loire. Les différences observées à l'aide d'Analyses Factorielles des Correspondances inter et intra classes démontrent l'intérêt d'une telle démarche mais aussi la nécessité de compléter cette étude par une analyse conjointe des facteurs de macro distribution. Les résultats obtenus nous permettent de concevoir différentes méthodes d'analyses pour une meilleure connaissance des hydrosystèmes potamiques.

Mots-clefs. - Loire, habitat, micro-distribution, macro-invertébrés. 
Abstract. - The identification of the main parameters controlling an ecological system is
necessary in order to understand its equilibrium and to help to its management. This
study, carried out by the "Plan Loire Grandeur Nature" program, shows a comparison
and a hierarchisation of the main ecological parameters controlling the micro-distribu-
tion of taxa at 2 stations on the Loire river : Bréhémont and La Charité-sur-Loire. Differ-
ences in the micro-distribution are observed at the 2 stations using a within and between
FCA analysis. An additional survey of macro-distribution parameters would be however
necessary to explain the differences observed in the micro distribution at the 2 stations.
This study also provides basis for the development of methods well adapted for the bio-
monitoring of large rivers.

Key-words. - Loire river, habitat, micro-distribution, macro-invertebrates.

\section{INTRODUCTION / PROBLÉMATIQUE}

Depuis quelques années, de nombreux dysfonctionnements, comme l'effondrement du pont Wilson à Tours en 1978, ont attiré l'attention des gestionnaires sur la nécessité d'intervenir sur l'hydrosystème ligérien (Dambre et al., 1993).

Les pouvoirs publics ont donc mis en oeuvre un vaste programme d'intervention, le Plan Loire Grandeur Nature, dont les principaux objectifs sont d'assurer la sécurité des biens et des personnes tout en maintenant ou en améliorant la qualité biologique de l'hydrosystème.

Cela nécessite de disposer de multiples informations sur l'ensemble du fleuve pour bien en comprendre le fonctionnement à différentes échelles spatiales et temporelles.

Dans ce contexte, il est indispensable de déterminer dans un complexe de facteurs, "dont les actions sur les espèces peuvent se situer à des échelles dimensionnelles très différentes, de la zone biogéographique aux micro-biotopes » (Ver- neaux, 1984), les principaux paramètres influant sur les biocénoses en place.

Un travail global a donc été entrepris sur différents compartiments biologiques (végétation, Cornier, 1998 ; invertébrés aquatiques, Bacchi, 2000) avec pour objectif de décrire les peuplements ligériens mais aussi de caractériser et de hiérarchiser les principaux facteurs structurants à l'échelle de la station (facteurs de macro-distribution) ou du micro-habitat (facteurs de micro-distribution).

C'est cette dernière approche, menée sur les peuplements d'invertébrés benthiques, qui est présentée ici.

Après avoir brièvement présenté les sites de Bréhémont et de La Charité-sur-Loire qui ont été choisis pour cette analyse et après avoir décrit le protocole expérimental utilisé, la première partie sera consacrée à la présentation très générale de la faune sur les deux stations ligériennes. Une attention toute particulière sera apportée au rôle joué par le substrat et la vitesse de courant comme le signalent de nombreux auteurs (Perrin, 
1978 ; Bournaud et al., 1986 ; Andersen et al., 1986 ; Cogerino, 1989).

Nous verrons ensuite, dans une deuxième partie, que l'utilisation des analyses inter ou intra-classes nous permettra de caractériser et de hiérarchiser différents paramètres de micro-distribution choisis parmi les plus représentatifs. Nous montrerons ainsi, par comparaison, des disparités intéressantes entre les deux sites analysés.

La conclusion nous permettra de souligner l'intérêt d'une telle approche pour une meilleure gestion des hydrosystèmes et d'insister sur la nécessité de généraliser cette étude aux paramètres de macro-distribution (Bacchi, in prep).

\section{MÉTHODOLOGIE}

\subsection{Choix des stations}

Dans le cadre de cette étude, deux sites ont été retenus pour échantillonnage. II s'agit d'un site en aval de La Charité-sur-Loire et d'un autre à Bréhémont (Fig. 1).

Ces sites ont été retenus car bien que distants de près de $300 \mathrm{~km}$, ils ont l'avantage de présenter des caractéristiques morphologiques et fonctionnelles similaires. De plus, les analyses typologiques de l'hydrosystème (Bacchi, 2000) ont montré que les peuplements présents sont semblables et qu'une comparaison de la structure des peuplements pouvait être établie sans que les différences

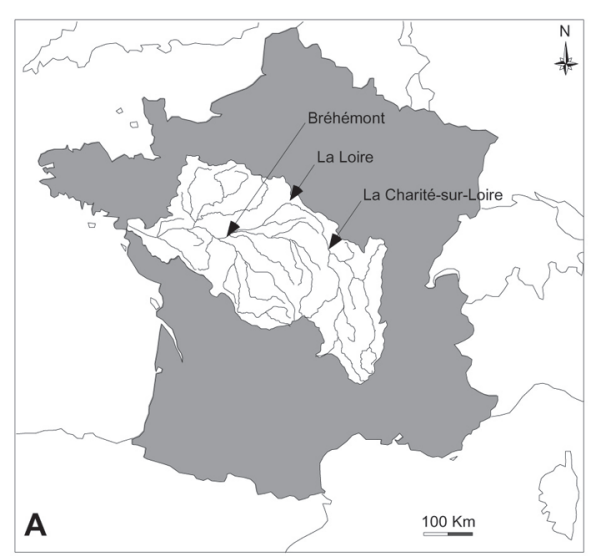

Fig. 1. - Situation du bassin versant de la Loire et localisation des sites de Bréhémont et La Charité-sur-Loire.

Fig. 1. - Location of the Loire river basin and of the 2 sampling sites Brehemont and La Charite sur Loire.

observées puissent être attribuées à la nature des taxons constituant ce peuplement. Notons toutefois que le site de Bréhémont subit des pressions anthropiques beaucoup plus fortes que le site de La Charité-surLoire avec une qualité d'eau très médiocre (Grosbois, 1998).

\subsection{Caractéristiques des stations}

Les principales caractéristiques des stations sont données dans le tableau I ci-dessous :

\subsubsection{Site de La Charité-sur-Loire.}

Ce site se situe à $40 \mathrm{~km}$ en aval du bec d'Allier. Quatre stations de prélèvement ont été implantées (Fig. 2 A) : 
Tableau I - Principales caractéristiques des sites de Loire analysés

Table I - Major characterictics of the 2 sampling sites

\begin{tabular}{|l|c|c|}
\hline & La Charité-sur-Loire & Bréhémont \\
\hline Distance à la source & $470 \mathrm{Km}$ & $761 \mathrm{Km}$ \\
\hline Pente moyenne du lit & $0,53 \mathrm{~m} / \mathrm{Km}$ & $0,42 \mathrm{~m} / \mathrm{Km}$ \\
\hline Altitude & 161 mètres & 35 mètres \\
\hline $\begin{array}{l}\text { Largeur moyenne du chenal principal } \\
\text { à l'étiage }\end{array}$ & 180 mètres & 260 mètres \\
\hline $\begin{array}{l}\text { Classe de qualité des eaux (Agence } \\
\text { de l'eau Loire Bretagne, 1994) }\end{array}$ & 2 & 3 \\
\hline
\end{tabular}

une station dans le lit vif, et trois dans des annexes hydrauliques. La station 4 est celle qui est déconnectée le plus rapidement du lit principal : cette rupture d'alimentation est effective pour un débit inférieur à $330 \mathrm{~m}^{3} / \mathrm{s}$. Les stations 2 et 3 sont restées faiblement alimentées par l'amont lors des différentes périodes d'échantillonnage.

\subsubsection{Site de Bréhémont}

Ce site se situe à $291 \mathrm{~km}$ en aval de La Charité-sur-Loire. Six stations de prélèvement ont été échantillonnées, trois stations dans le lit vif et trois autres dans les annexes hydrauliques (Fig. 2, B). La station 2 se déconnecte du lit de la Loire assez rapidement dans l'année pour un dé-
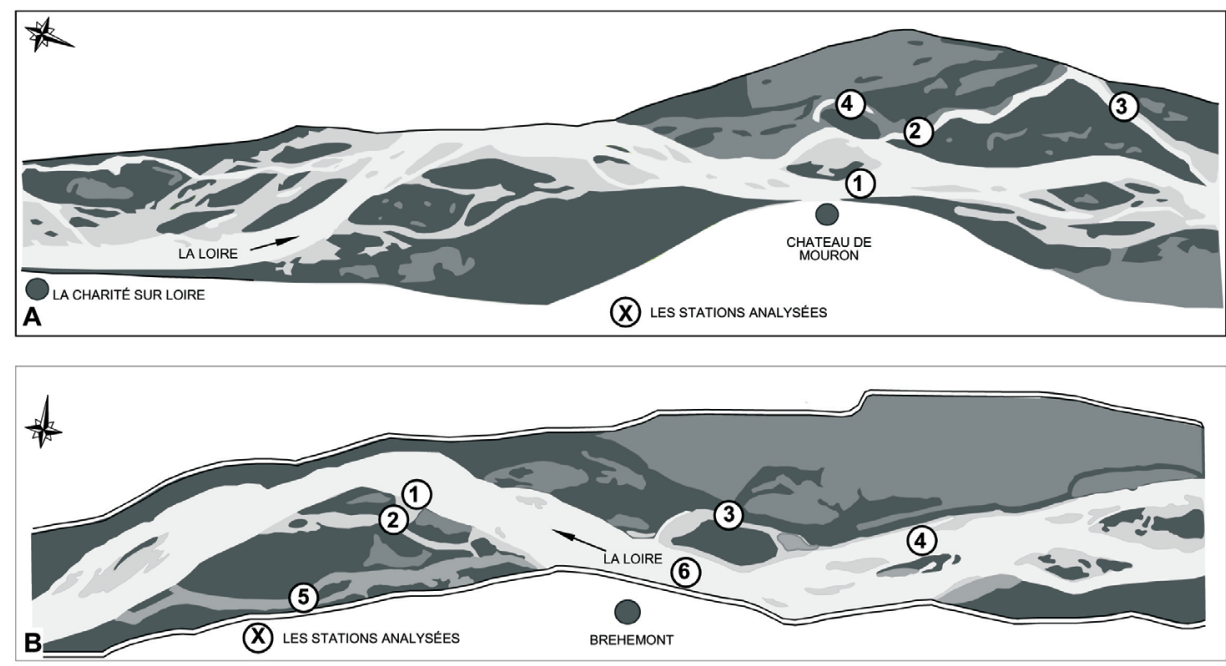

Fig. 2. - Présentation des sites.

A : Présentation du site de La Charité-sur-Loire et localisation des stations analysées.

B : Présentation du site de Bréhémont et localisation des stations analysées.

Fig. 2. - Location of the stations A- at La Charité-sur-loire, and B- at Bréhémont 
bit d'environ $530 \mathrm{~m}^{3} / \mathrm{s}$ et le temps total d'alimentation de ce bras a été de 55 jours en 1996 ; la station 3 est implantée dans un bras encore actif puisqu'il a été alimenté 212 jours au total en 1996. La station 5 est la plus souvent déconnectée du lit principal. Son alimentation n'est possible que lors de débits supérieurs à $550 \mathrm{~m}^{3} / \mathrm{s}$.

\section{3 Échantillonnage de la macrofaune benthique}

Les invertébrés benthiques n'ont été que peu étudiés sur la Loire. En effet, hormis le suivi régulier des centrales nucléaires, on ne recense que quelques études sur la présence d'espèces particulières (Chovet et al., 1984 et 1994 ; Lécureuil et al., 1983 et 1984 ; Garcia et al., 1999a et 1999b ; Garcia, 1994) et peu d'études globales (Bacchi, 2000 ; Ivol et al., 1997 ; Guinand et al., 1998) qui esquissent la structure typologique du fleuve.

Si les techniques utilisées dans les petits cours d'eau sont maintenant bien connues et maîtrisées, l'analyse des mosaïques d'habitats des grands cours d'eau pose, à bien des égards, des problèmes méthodologiques liés à la taille mais aussi au mode de fonctionnement des systèmes analysés.

Les données bibliographiques traitant de la structuration des micro-habitats en grands cours d'eau ne sont pas nombreuses. On peut attribuer ce fait à la difficulté que pose l'échantillonnage des fleuves. C'est à partir de travaux comme ceux de Cogerino
(1989) que nous avons élaboré notre plan d'échantillonnage. La procédure de Bournaud et al. (1986) reprend les travaux de Favet (1981), Jenkins et al. (1984).)

Après avoir testé différentes méthodes d'échantillonnage (Bacchi, 2000) nous avons opté pour un échantillonnage au filet Surber de maille de $500 \mu \mathrm{m}$ en l'adaptant à nos besoins (Surber classique en rive et au centre du chenal, Surber emmanché et manipulé par deux personnes dans un bateau).

\subsubsection{Les substrats analysés}

Deux principaux types de substrats ont été retenus: les substrats « organiques » et les substrats minéraux. Quatorze types ont été reconnus comme étant les plus caractéristiques de la Loire et retenus pour la suite de ce travail. Une description de ces substrats est donnée dans le tableau II.

La vitesse de courant a été mesurée au niveau de chaque prélèvement, à $10 \mathrm{~cm}$ au dessus du substrat échantillonné, pour caractériser au mieux l'ambiance du micro-habitat vis-à-vis de ce paramètre. Cette mesure de vitesse a été faite avec un micro-moulinet O.T.T. (comptage sur 30 secondes et valeur finale obtenue en prenant la valeur moyenne de deux prises de mesures). La profondeur d'échantillonnage dépendait en partie des variations de hauteurs d'eau observées sur le site les jours précédents l'échantillonnage. Nous n'avons échantillonné que des subs- 
Tableau II. - Les différents types de substrats : principales caractéristiques

Table II. - Main characteristics of the different types of substrats

\begin{tabular}{|l|l|}
\hline Type de substrat & Caractéristiques \\
\hline Substrats organiques & \\
\hline Algues filamenteuses & - \\
\hline Racines & Chevelu dense \\
\hline Végétaux émergents & $\begin{array}{l}\text { Hélophytes ou végétaux terrestres } \\
\text { à moitié immergés }\end{array}$ \\
\hline Végétaux immergés & Hydrophytes ou végétaux submergés \\
\hline Bois & Troncs d'arbres ou branches immergés \\
\hline Substrat fin à dominante organique ('vases') & \\
\hline Débris organiques & Feuilles, brindilles \\
\hline Substrats minéraux & \\
\hline Sables & Diamètre $<2 \mathrm{~mm}$ \\
\hline Graviers & $2 \mathrm{~mm}-3 \mathrm{~cm}$ \\
\hline Galets & $3 \mathrm{~cm}-15 \mathrm{~cm}$ \\
\hline Blocs & Diamètre $>15 \mathrm{~cm}$ \\
\hline Graviers-galets & $2 \mathrm{~mm}-15 \mathrm{~cm}$ \\
\hline Sables-graviers & $<3 \mathrm{~cm}$ \\
\hline Sables-graviers-galets & $<15 \mathrm{~cm}$ \\
\hline
\end{tabular}

trats immergés au moins 15 jours avant le prélèvement.

\subsubsection{Traitement des échantillons}

Les prélèvements ont été immédiatement formolés sur le terrain (formol en solution à $8 \%$ pour la conservation). La faune a été triée en laboratoire après lavage des prélèvements sur un tamis de diamètre de maille $500 \mu \mathrm{m}$. L'ensemble de la faune a été collecté.

\subsubsection{Unités taxinomiques retenues}

Les taxons collectés ont été déterminés à différents niveaux taxinomiques suivant les connaissances précédemment acquises par d'autres auteurs sur la faune ligérienne. Si certains groupes ont pu être assez facilement déterminés à l'espèce (Plécoptères, Ephémères et Trichoptères) ou au genre (Coléoptères et Crustacés), la méconnaissance actuelle des autres peuplements ligériens (Diptères Odonates, Oligochètes, Achètes...) nous a contraint pour ces groupes, et compte tenu du nombre très important de prélèvements réalisés, à ne retenir que le niveau taxinomique de l'ordre ou de la famille.

Les résultats présentés ici sont issus de données faunistiques genre/famille (voir tableau des limites de détermination en annexe I). Des travaux ont été réalisés sur un moins grand nombre d'échantillons à l'espèce; ils corroborent les résultats présentés ici (Bacchi, 2000). 


\subsection{Périodes d'échantillonnage}

Le site de Bréhémont a été échantillonné tous les quinze jours au cours du printemps et de l'été 1996 (du début mars à fin août) et tous les mois de janvier à février 1996 et de septembre 1996 à janvier 1997 (Fig. 3). Le site de La Charité-sur-Loire a été échantillonné avec une fréquence mensuelle et uniquement pendant les périodes printanière et estivale (échantillonnage le 28 avril, 25 mai, 19 juin et 19 juillet 1996). Pour chaque date, un maximum de couples substrat-vitesse ont été échantilIonnés.

Le nombre total de prélèvements s'élève à 738 sur le site de Bréhémont et 204 sur le site de La Charitésur-Loire, soit un total de 942 prélèvements. Le site de La Charité-surLoire n'a été échantillonné que pendant la période de développement maximum de la faune (d'avril à août) car il n'était pas possible de réaliser le même effort d'échantillonnage sur les deux sites.

\section{LES RÉSULTATS}

\subsection{Observations générales}

Le tableau III ci-dessous résume les différentes caractéristiques de la macrofaune benthique analysée sur les sites de La Charité-sur-Loire et Bréhémont.

On remarque que les peuplements des deux sites se caractérisent par un nombre très important d'Oligochètes et de diptères Chironomidés. Ceux-ci représentent $56,5 \%$ et $77 \%$ du nombre total d'individus échantillonnés. L'abondance moyenne relativement faible par prélèvement (936 individus pour $1 / 20^{\text {ème }}$ de $\mathrm{m}^{2}$ ) est due au fait que tous les habitats ont été prospectés systématiquement, y compris ceux qui étaient les moins susceptibles d'héberger une faune

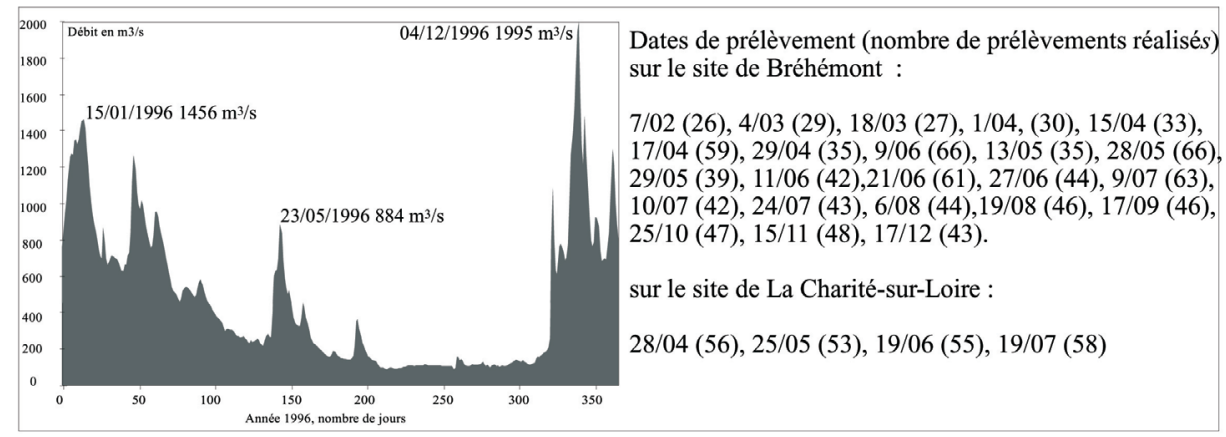

Fig. 3. - Variation de débit au cours de l'année 1996 sur le site de Bréhémont (dates et nombres de prélèvements réalisés au cours de l'année sur les deux sites).

Fig. 3. - Hydrological variations at Brehemont in 1996. Dates and numbers of samples at the 2 sampling sites. 
Tableau III. - Données générales sur les peuplements échantillonnés sur les sites de Bréhémont et la Charité-sur-Loire

Table III. - General data of the macrobenthic populations at the 2 sampling sites

\begin{tabular}{|l|c|c|c|}
\hline & $\begin{array}{c}\text { La Charité-sur- } \\
\text { Loire }\end{array}$ & Bréhémont & $\begin{array}{c}\text { Total et } \\
\text { moyenne }\end{array}$ \\
\hline Nombre d'échantillons $\left(1 / 20 \mathrm{~m}^{2}\right)$ & 204 & 738 & 942 \\
\hline Abondance totale & 176588 & 705578 & 882166 \\
\hline Abondance moyenne par prélèvement & 866 & 956 & 936 \\
\hline $\begin{array}{l}\text { Pourcentage d'Oligochètes et de } \\
\text { Chironomidés }\end{array}$ & $56.5 \%$ & $77 \%$ & $72.9 \%$ \\
\hline Nombre total de taxons (genre-famille) & 93 & 136 & 139 \\
\hline $\begin{array}{l}\text { Nombre de taxons échantillonnés dans } \\
\text { le lit vif }\end{array}$ & $63(67.7 \%)$ & $99(72.8 \%)$ & - \\
\hline $\begin{array}{l}\text { Nombre de taxons spécifiques } \\
\text { du lit vif }\end{array}$ & $3(3.2 \%)$ & $13(9.5 \%)$ & - \\
\hline $\begin{array}{l}\text { Nombre de taxons échantillonnés dans } \\
\text { les annexes hydrauliques }\end{array}$ & $89(95.7 \%)$ & $121(89.0 \%)$ & - \\
\hline $\begin{array}{l}\text { Nombre de taxons spécifiques } \\
\text { aux annexes hydrauliques }\end{array}$ & $29(31.2 \%)$ & $37(27.2 \%)$ & - \\
\hline
\end{tabular}

abondante et diversifiée que constituent les sédiments les plus instables (sable en milieu de chenal par exemple).

Le nombre total de taxons (genrefamille) est élevé sur les deux stations puisque l'on en recense 93 à $\mathrm{La}$ Charité-sur-Loire et 136 à Bréhémont.

\subsubsection{Distribution spatiale et temporelle}

D'une façon générale, les taxons du lit vif représentent la plus grande partie du peuplement $(67,7 \%$ sur La Charité et $72,8 \%$ sur Bréhémont). Toutefois, les annexes hydrauliques peuvent accueillir un pourcentage encore plus élevé de taxons (respectivement $95,7 \%$ et $89,0 \%$ ). Ces fortes valeurs s'expliquent par les caractéristiques particulières de ces milieux qui hébergent des taxons spécifiques que l'on ne retrouve pas dans le lit vif, $29(31,2 \%)$ sur La Charité-sur-Loire et $37(27,2 \%)$ sur Bréhémont. De plus, après des crues importantes, de nombreux taxons du lit vif dérivent dans ces bras et participent à l'augmentation de la biodiversité observée.

La répartition spatiale et temporelle des taxons selon la méthode de Guidicelli (Guidicelli, 1968) sur les deux sites analysés est donnée dans la figure 4 montrant que les taxons fondamentaux et constants sont sensiblement les mêmes sur les deux sites. Toutefois, de nombreux taxons accessoires ou sporadiques n'ont été inventoriés que sur le site de Bréhémont. Cela est dû, notamment, à la présence de nombreux coléoptères et hétéroptères que l'on retrouve sur 


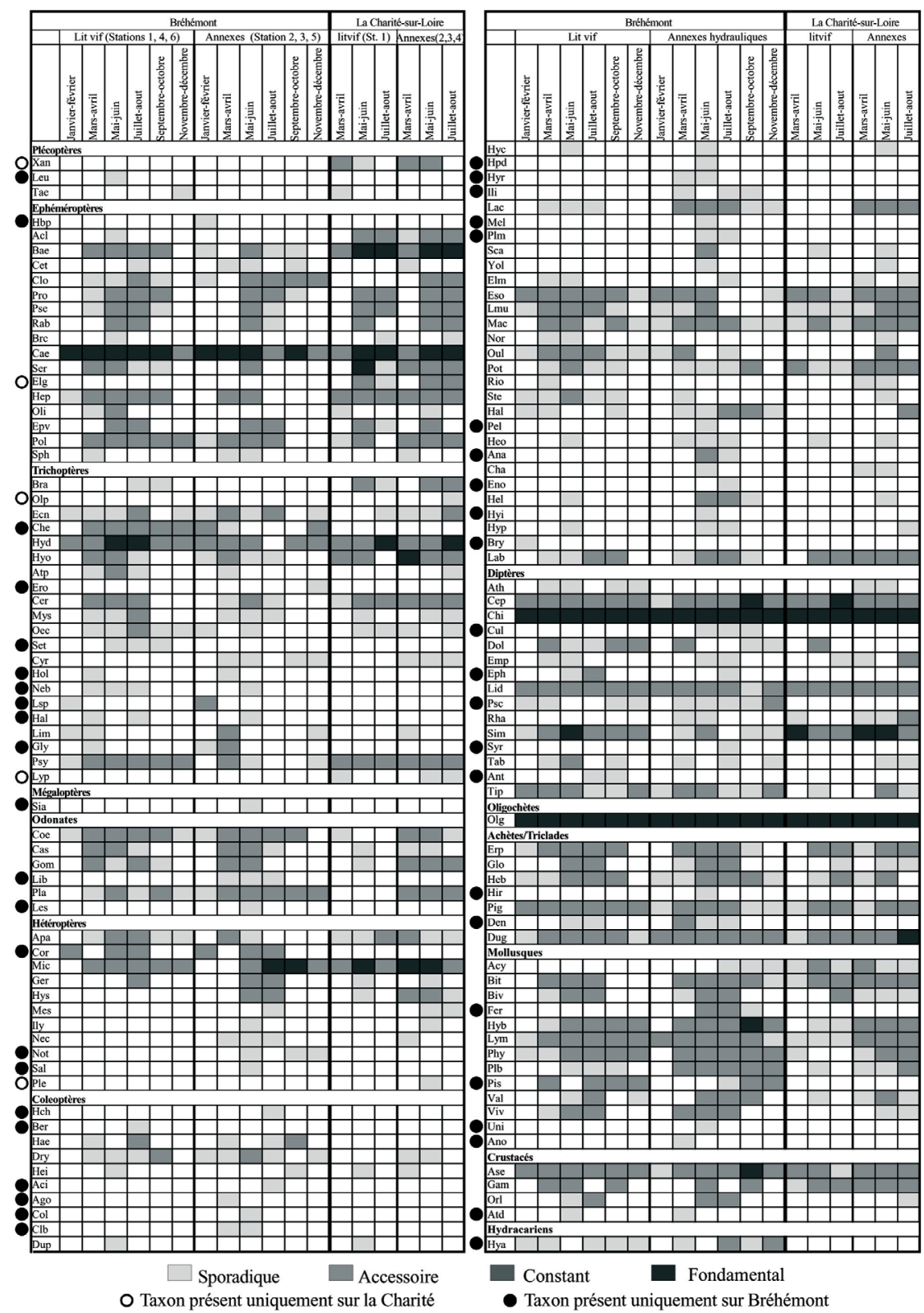

Fig. 4. - Répartition des taxons selon la méthode de Guidicelli (1968) en fonction des stations et des dates d'échantillonnage sur les sites de Bréhémont et La Charité-sur-Loire (année 1996).

Fig. 4. - Distribution of fauna according to the Guidicelli method (1968) versus stations and sampling dates at the 2 sampling sites in 1996 
les annexes hydrauliques du site de Bréhémont.

\subsubsection{Distribution taxinomique par type de substrat}

La relation taxon/substrat a déjà fait l'objet de très nombreux travaux sur les cours d'eau. Outre des articles très généraux (Cummins et al., 1969 ; Hynes, 1970 ; Prodon, 1976), la bibliographie est riche d'un très grand nombre de travaux qui font référence à l'utilisation des substrats par différentes communautés macrobenthiques en petits ou grands cours d'eau (Auct. in Cogerino, 1989; Beisel, 1996).

L'hydrosystème ligérien présentant de nombreuses originalités, il paraît important de préciser ici l'utilisation des différents habitats par les invertébrés aquatiques.

La figure 5 illustre, pour les deux sites analysés, la distribution des différents taxons dans les divers substrats. Le nombre de prélèvements par substrat étant très variable, les données sont présentées sous forme de courbes de richesse cumulée calculées à partir d'un ré-échantillonnage aléatoire dans l'ensemble des relevés (Ferry \& Frochot, 1970 ; Chessel et al., 1993). Cette technique permet de comparer, pour un même nombre d'échantillons, la variété taxinomique et rend aussi possible l'évaluation de la stabilité de l'échantillonnage.

La forme des courbes de richesse cumulée (Fig. 5) montre que malgré le nombre très important de prélèvements réalisés, l'échantillonnage n'a pas permis la capture de tous les taxons. Ces résultats soulignent la difficulté de l'échantillonnage de la macrofaune benthique en grands cours d'eau et posent la question de l'utilisation des protocoles d'échantilIonnage normalisés à 8 prélèvements (type IBGN) pour des analyses particulières de l'hydrosystème. Si ceuxci permettent la détermination de la qualité générale du milieu dans un cadre référentiel bien défini, Verneaux a déjà souligné leur inadéquation à des études d'impact relevant de la biocénotique approfondie (Verneaux, 1994) alors que seule une faible partie de la richesse taxinomique globale est collectée. Notons toutefois que la très grande similitude dans la hiérarchie des richesses taxinomiques par substrat sur les deux sites nous laisse envisager la mise au point d'un protocole d'échantillonnage qui pourrait être commun à l'ensemble de l'hydrosystème ligérien pour mener ce type d'étude (Bacchi, in prép.).

On remarque sur les deux sites une organisation très semblable de la richesse taxinomique (Fig. 5). La richesse est maximum pour les racines d'arbres, les végétaux émergents et immergés, les bois et les blocs, mais bien moindre pour les autres substrats : sables, graviers ou galets mélangés ou non aux substrats de plus faible granularité. Cette différence s'explique par une plus grande complexité spatiale source de très nombreux abris, et par la relative stabilité des substrats végétaux qui permet, même lors de contraintes hydrauli- 

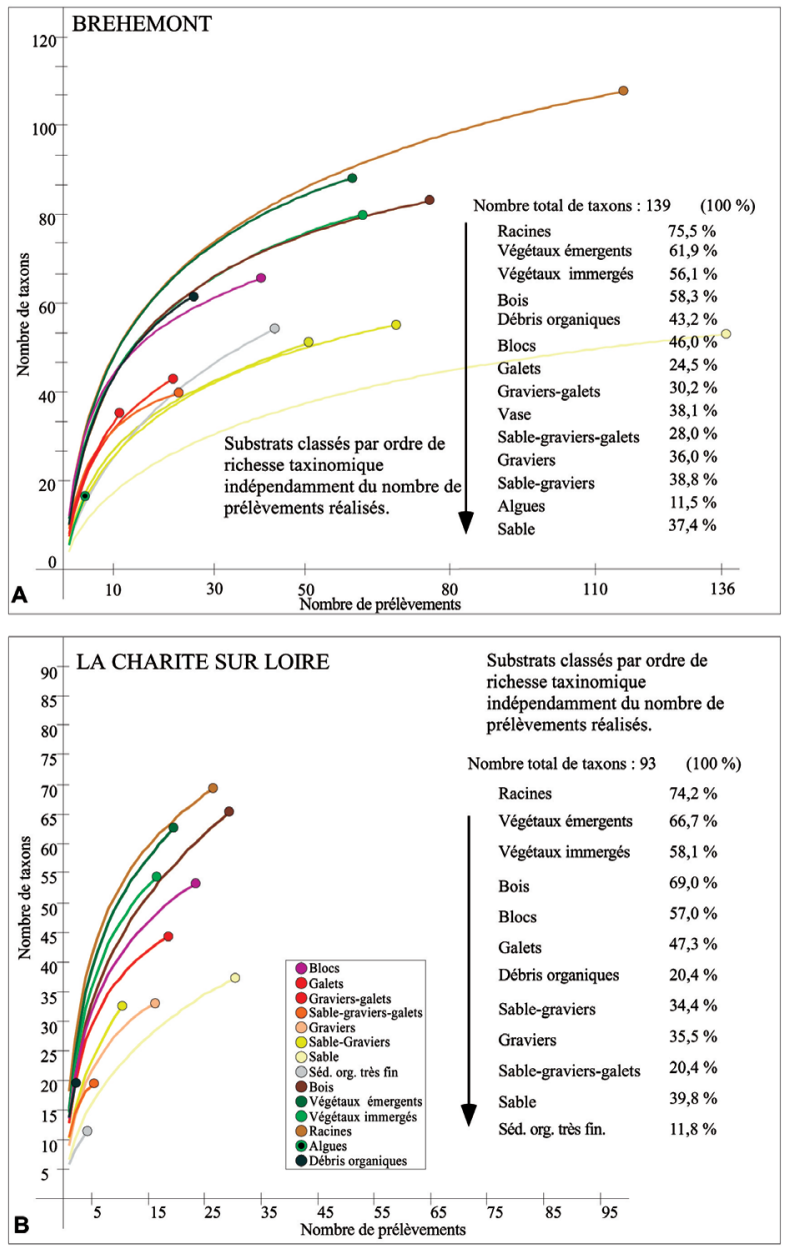

Fig. 5. - Courbes des richesses cumulées des substrats analysés sur les sites de Bréhémont $(A)$ et de La Charité-sur-Loire (B) en 1996

Fig. 5. - Richness cumulated curve : (A) Bréhémont, (B) La Charité-sur-Loire

ques fortes, le maintien d'un cortège faunistique diversifié.

La richesse taxinomique est aussi influencée par les apports trophiques directs ou indirects dus aux substrats organiques (consommation de la matière organique ou ingestion de micro-organismes liés à sa dégradation comme les bactéries ou les champignons ; Iversen, 1974 ; Lapchin, 1977 ; Becker 1994). Cela explique le nombre important de taxons recensés dans les débris organiques et dans les sédiments minéraux très fins riches en matière organique («vases »). 
Ces résultats ne correspondent pas aux travaux de Cogérino (1989) sur le Rhône. En effet, les substrats racines et hélophytes qui apparaissent très importants en Loire car porteurs d'une très forte biodiversité, sont caractérisés sur le Rhône par une richesse relative bien inférieure à celle des bryophytes, algues, blocs et débris organiques. Cela souligne les fortes disparités qui existent entre les hydrosystèmes fluviaux et l'impossibilité de généraliser, sans précaution, les résultats obtenus.

\subsection{Analyse des facteurs de micro-distribution sur le site de Bréhémont}

Les facteurs de micro-distribution ont été peu étudiés en grands cours d'eau ou alors, comme sur le Rhône, de façon très partielle avec la seule analyse des habitats rivulaires (Bournaud et al., 1986 ; Cogerino, 1989). Cette méconnaissance tient surtout dans la difficulté d'échantillonnage de tels milieux qui a conduit de nombreux auteurs à échantillonner avec des dragues et/ou des substrats artificiels qui ne permettent pas nécessairement une analyse des habitats.

Les variables présentées ici sont celles classiquement utilisées dans l'étude des habitats aquatiques: la nature du substrat, la vitesse de courant, le couple substrat/vitesse, la profondeur. Après avoir caractérisé ces différents facteurs de micro-distribution sur le site de Bréhémont, nous utiliserons les Analyses Factorielles des Correspondances inter et intra classes pour hiérarchiser ces facteurs entre eux.

\subsubsection{Caractérisation des facteurs de micro-distribution}

La caractérisation des facteurs de micro-distribution est possible par l'utilisation des Analyses Factorielles des Correspondances inter et intraclasses. En effet, celles-ci permettent d'identifier différentes sources de variabilité, par la prise en compte (analyse inter-classes) ou l'élimination (analyse intra-classes) d'un facteur donné. Cette technique est basée sur la modification du tableau d'entrée (Doledec et al., 1989). L'analyse inter-classes consiste à optimiser la dispersion dans le plan factoriel des centres de gravité des profils moyens de la variable testée. L'analyse intraclasses, qui est une approche complémentaire de la précédente, permet d'étudier la structure résiduelle après élimination de l'effet d'une variable en positionnant les centres de gravité de la variable analysée au centre des axes du plan factoriel.

La matrice faunistique utilisée, après élimination des taxons accidentels, est une matrice 733 lignes " prélèvements " et 105 colonnes " taxons » (genre/famille). Les abondances brutes sont transformées par la fonction $y=\log (x+1)$ et les matrices traitées par Analyse Factorielle des Correspondances inter et intra classes.

Pour chaque variable un test de permutation multivarié est réalisé pour déterminer le degré de signification des paramètres analysés. Seules 
les variables dont les tests sont significatifs ont été retenues et décrites par l'analyse discriminante.

La figure 6 illustre la projection des variables profondeur (A) et vitesse de courant (B), et la figure 7 la projection des variables substrat $(C)$ et substratvitesse (D) dans le plan F1/F2 des AFC inter-variables pour chacun des paramètres cités. Le premier graphique (a) positionne le centre d'inertie de chaque variable dans l'analyse inter-classes, le deuxième (b) illustre la position moyenne de chaque taxon. Un tableau de synthèse des abréviations utilisées est donné en annexe I.

\subsection{1.a La profondeur}

$\mathrm{Ce}$ paramètre n'explique qu'une très faible part de la variabilité totale (Fig. 6, Aa). L'axe F1 porte l'essentiel de l'information (59\%) et discrimine la faune des profondeurs de plus de 1 mètre. Les taxons placés au centre des axes comme Hydropsyche, Chironomidae, Caenis, Baetis, prouvent leur indépendance vis-à-vis de la profondeur (Fig. 6, Ab). D'autres taxons tels que les Oligochètes et les Ceratopogonidae apparaissent comme plus structurants. Ils préfèrent surtout les sables du milieu de chenal, ce qui correspond aux secteurs les plus profonds échantillonnés.

\subsection{1.b La vitesse de courant}

Tout comme pour la profondeur, c'est l'axe F1 qui porte l'essentiel de l'information avec $61 \%$ de l'inertie totale expliquée (Fig. 6, Ba). Les vitesses de 0 à $0,7 \mathrm{~m} / \mathrm{s}$ montrent une progression logique sur l'axe F1 qui traduit une différenciation continue des taxons en fonction de ce facteur. Les taxons liés aux faibles vitesses de courant (Fig. 6, Bb) sont essentiellement ceux des annexes hydrauliques, des Coléoptères ou certains Ephéméroptères (Cloeon) alors que l'on retrouve à l'opposé sur le même axe des taxons rhéophiles comme Oligoneuriella, Cheumatopsyche, Rabtobaetopus, Hydropsyche, Baetis ou Ephoron. Les vitesses supérieures à $0,7 \mathrm{~m} / \mathrm{s}$ se différencient nettement sur l'axe F2 ce qui peut s'expliquer par leur effet perturbateur ne permettant pas la structuration du cortège faunistique sur de nombreux substrats instables.

\subsection{1.c Le substrat}

La structure obtenue (Fig. 7, Ca) différencie très nettement les substrats organiques des minéraux. Si les premiers semblent héberger les mêmes taxons puisque la dispersion des centres d'inertie dans le plan factoriel est faible, il n'en va pas de même pour les seconds qui s'identifient très clairement en fonction de la granularité. On observe ainsi un très net gradient entre les sédiments sableux très organiques, les sables, les graviers, les galets et les blocs. II est intéressant de constater que la faune semble davantage associée à la fraction granulométrique la plus grossière du sédiment. En effet, les centres d'inertie des substrats hétérométriques identifiés par un mélange, sables-galets, sables-graviersgalets ou sables-graviers, sont voisins de ceux des substrats dont la 

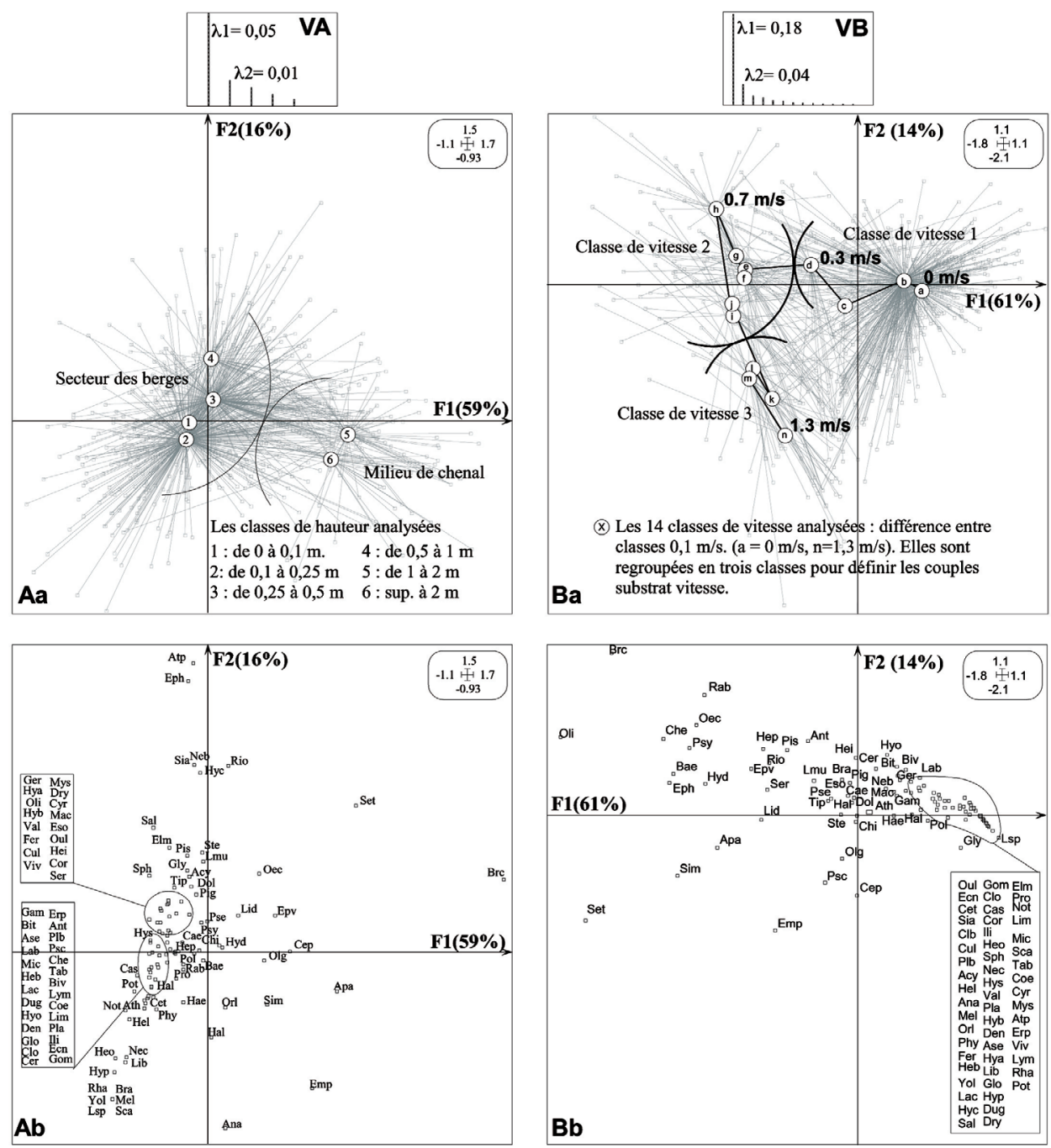

Fig. 6 
composition granulométrique homométrique est celle des éléments les plus grossiers: les galets et les graviers.

L'analyse faunistique (Fig. 7, Cb) fait ressortir les taxons électifs des substrats organiques (Asellidae, Gammaridae) de ceux qui préfèrent les substrats minéraux grossiers (Cheumatopsyche ou Heptagenia), les substrats à granularité moyenne (Ephoron), ou les sédiments sableux (Ceratopogonidae, Oligochètes). Certains taxons placés près du centre des axes montrent leur indépendance visà-vis de la nature du substrat (Caenis, Piscicola,...).

\subsection{1.d Le couple substrat-vitesse}

Afin d'établir les couples substratvitesse, les vitesses ont été regroupées en trois classes (Fig. 6, Ba). II y a là une légère perte d'information, nécessaire cependant pour limiter le nombre de couples substrat-vitesse possibles.
L'analyse de la figure $7 \mathrm{Da}$ fait ressortir une image plus complexe, très fortement structurée par les vitesses de courant. On observe :

- pour des vitesses inférieures à $0,2 \mathrm{~m} / \mathrm{s}$ une séparation des substrats selon le schéma évoqué précédemment, c'est-à-dire avec une discrimination des substrats organiques et minéraux. Les blocs situés en courant lent s'isolent des autres types de substrat.

- pour des vitesses moyennes ou fortes $(>0,2 \mathrm{~m} / \mathrm{s})$, un regroupement de la presque totalité des couples substrat-vitesse qui démontre la sélection de la faune par les fortes vitesses de courant quelle que soit la nature du substrat. Une telle structure prouve l'existence de conditions limites qui imposent aux invertébrés benthiques un habitat particulier, de préférence lié à la vitesse de courant. $\mathrm{Ce}$ paramètre mésologique serait, sur le site de Bréhémont, un facteur de survie.

Fig. 6. - Premier plan factoriel des AFC inter-classes profondeur et inter-classes vitesse du tableau (733 lignes prélèvements, 105 colonnes taxons) des prélèvements réalisés sur le site de Bréhémont.

VA: Histogramme des valeurs propres AFC inter-profondeur.

VB : Histogramme des valeurs propres de l'AFC inter-vitesse.

Aa : Position des centres d'inertie des profondeurs de prélèvement par AFC inter-profondeur.

Ab : Plan factoriel des taxons dans l'AFC inter-profondeur.

Ba : Position des centres d'inertie des vitesses dans l'AFC inter-vitesse.

Bb : Plan factoriel des taxons dans l'AFC inter-vitesse.

Fig. 6. - F1-F2 factorial plane of between depth and current velocity COA analysis Projection of variables and taxa on the first factorial plane. Site of Bréhémont (1996).

VA : Eigenvalue diagram of between depth COA analysis.

VB : Eigenvalue diagram of between current velocity COA analysis.

Aa : Results of the between-depth COA analysis. Factorial plane (1-2) of samples separated by depth

$\mathbf{A b}$ : Results of the between-depth COA analysis. Factorial plane (1-2) of fauna.

$\mathrm{Ba}$ : Results of the between current velocity COA analysis. Factorial plane (1-2) of samples separated by current velocity.

Bb : Results of the between current velocity COA analysis. Factorial plane (1-2) of fauna. 


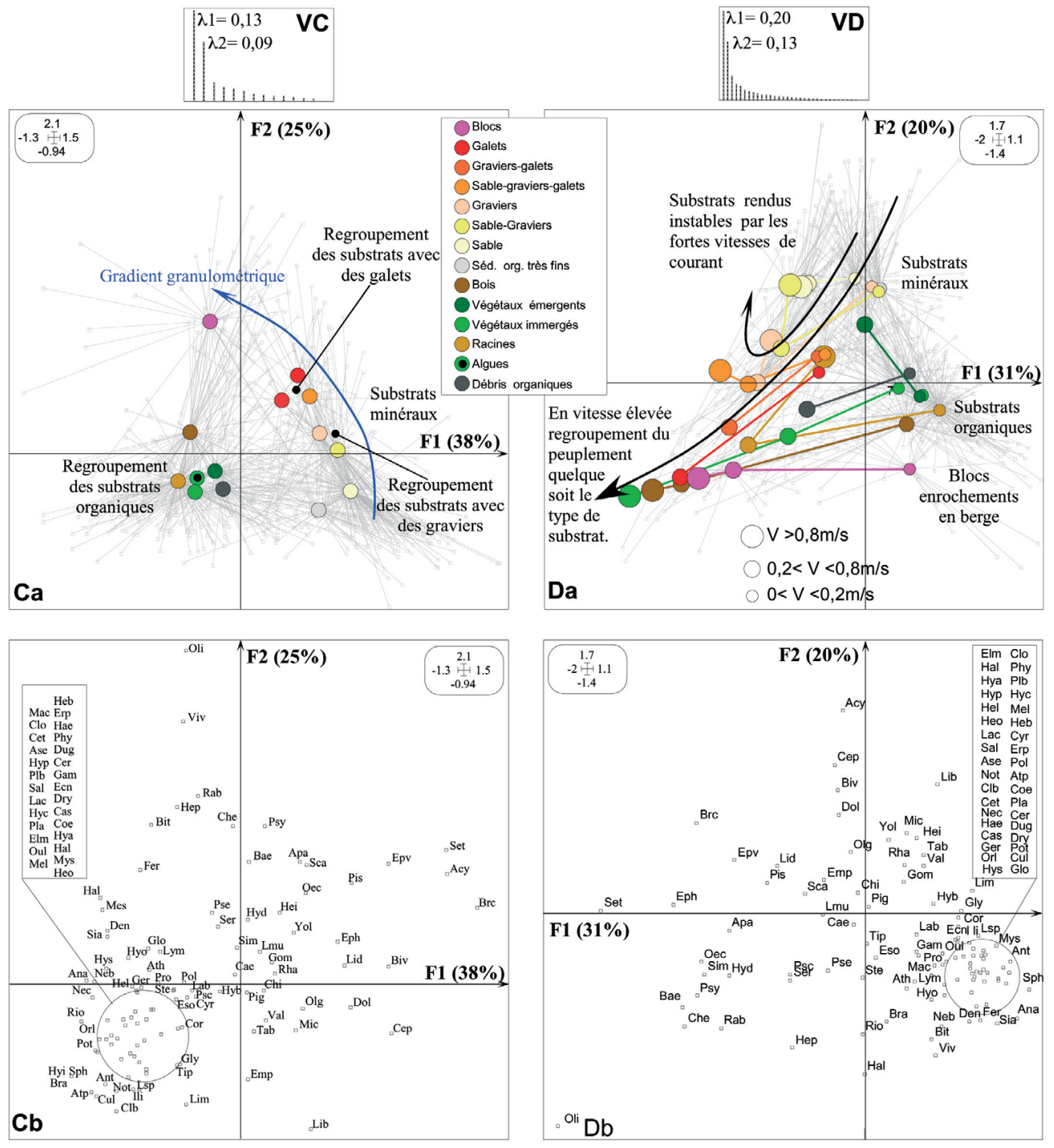

Fig. 7 
Le regroupement des couples substrat-vitesse n'est toutefois pas total puisque les substrats rendus instables par les fortes vitesses de courant (sables, sables-graviers, graviers, sables-graviers-galets) ne suivent pas le schéma précédemment défini, ces vitesses ne permettant pas l'implantation d'un cortège faunistique sur ces substrats.

La figure $7 \mathrm{Db}$ permet de caractériser les taxons très liés aux importantes vitesses de courant (quelle que soit la nature du support). On note la position très particulière d'Oligoneuriella, très fortement discriminante, et d'un groupe de taxons très liés aux vitesses rapides (Hydropsyche, Oecetis, Simuliidae, Psychomyia, Baetis, Cheumatopsyche, et Rabtobaetopus).

\subsubsection{Hiérarchisation des facteurs de micro-distribution}

La hiérarchisation des paramètres structurants de la macrofaune est appréciée grâce aux Analyses Factorielles des Correspondances inter et intra-classes. Celles-ci permettent en effet d'estimer l'importance d'un facteur de micro-distribution en comparant la position relative des premières valeurs propres de chacune des analyses par rapport à la première valeur propre de l'analyse globale (Degiorgi, 1994 ; Verneaux, 1996). Cette hiérarchisation a été conduite sur le site de Bréhémont à deux niveaux : globalement et par saison.

\subsection{2. a Analyse de la structure globale}

Les paramètres structurants de la macrofaune (Tableau IV), caractérisés par les premières valeurs propres des Analyses Factorielles des Correspondances inter et intra-classes, permettent de faire les observations suivantes:

Fig. 7. - Premier plan factoriel des AFC inter-classes substrat et inter-classes substrat-vitesse du tableau (733 lignes prélèvements, 105 colonnes taxons) des prélèvements réalisés sur le site de Bréhémont en 1996.

VC : Histogramme des valeurs propres AFC inter-substrat.

VE : Histogramme des valeurs propres de l'AFC inter substrat-vitesse.

$\mathrm{Ca}$ : Position des centres d'inertie des substrats par AFC inter-substrat.

$\mathbf{C b}$ : Plan factoriel des taxons dans l'AFC inter-substrat.

Da : Position des centres d'inertie des substrats-vitesses dans l'AFC inter substrat-vitesse.

Db : Plan factoriel des taxons dans l'AFC inter substrat-vitesse.

Fig. 7. - F1-F2 factorial plane of between substrats and substrat/current velocity COA analysis Projection of variables and taxa on the first factorial plane. Site of Bréhémont (1996).

VC : Eigenvalue diagram of between substrats COA analysis.

VE : Eigenvalue diagram of between substrat/current velocity $C O A$ analysis.

$\mathrm{Ca}$ : Results of the between-substrats COA analysis. Factorial plane (1-2) of samples separated by substrats

$\mathrm{Cb}$ : Results of the between-substrat COA analysis. Factorial plane (1-2) of fauna.

$\mathrm{Da}$ : Results of the between substrat/current velocity COA analysis. Factorial plane (1-2) of samples separated by current velocity.

Db : Results of the between substrat/current velocity COA analysis. Factorial plane (1-2) of fauna. 
Tableau IV. - Premières valeurs propres $(\lambda 1)$ des AFC simples inter et intra classes réalisées à partir des relevés faunistiques sur le site de Bréhémont au cours de l'année 1996.

Table IV. - First eigenvalue $(\lambda 1)$ of COA and between and within COA analysis : Site of Bréhémont (1996)

\begin{tabular}{|l|c|c|}
\hline Variables & Analyse inter-classes & Analyse intra-classes \\
\hline AFC simple & \multicolumn{2}{|c|}{$\lambda 1=0,36$} \\
\hline AFC inter substrat/vitesse & 0,21 & 0,20 \\
\hline AFC inter vitesse & 0,19 & 0,25 \\
\hline AFC inter substrat & 0,14 & 0,31 \\
\hline AFC inter profondeur & 0,05 & 0,34 \\
\hline
\end{tabular}

Les paramètres de micro-distribution expliquent une part importante de la variabilité globale observée sur le site de Bréhémont. Le substrat ou la vitesse apparaissent comme les principaux facteurs de structuration des peuplements, ce qui confirme les travaux de très nombreux auteurs. Toutefois, on remarque la part prépondérante prise par la vitesse de courant $(\lambda 1=0,19)$ sur le substrat $(\lambda 1=0,14)$ ce qui confirme, sur ce site, le rôle de ce paramètre dans la distribution de la faune. La profondeur $(\lambda 1=0,05)$, bien qu'étant significative, n'exprime qu'une très faible part de la variabilité globale.

\subsection{2.b Evolution diachronique des facteurs de micro-distribution}

L'analyse a été conduite à partir de la matrice genre/famille (733 lignes prélèvements et 105 colonnes taxons) scindée en quatre unités regroupant des cortèges taxinomiques différents. La première regroupe les prélèvements d'octobre à février (97 lignes prélèvements, 59 colonnes taxons), la deuxième ceux de mars-avril (184 lignes prélèvements, 72 colonnes taxons), la troisième ceux de mai- juin (275 lignes prélèvements, 86 coIonnes taxons), et la quatrième ceux de juillet à septembre (177 lignes prélèvements, 79 colonnes taxons). Les données ont été transformées par la fonction $y=\log (x+1)$ et traitées par Analyse Factorielle des Correspondances inter-classes.

La comparaison des premières valeurs propres des AFC inter-variables (Tableaux V et VI) permet d'observer que le couple substrat-vitesse apparaît toujours comme l'élément principal d'organisation de la faune sur le site de Bréhémont. Dans ce couple substrat-vitesse, la vitesse de courant apparaît, toute date confondue, comme plus structurante que le substrat. La position en rive ou en milieu de chenal, qui traduit d'une certaine manière la profondeur, n'apparaît que peu discriminante. On remarque dans le tableau VI que la part relative prise dans la structuration des peuplements est maximale de juillet à septembre mais aussi en mars-avril. Les faibles valeurs enregistrées d'octobre à février et en mai juin sont dues à de fortes crues qui ont très fortement modifié la structure des peuplements en place. 
Tableau V. - Premières valeurs propres $(\lambda 1)$ des AFC simples et inter classes réalisées à partir des relevés faunistiques par date de prélèvement sur le site de Bréhémont au cours de l'année 1996 Table V. - First eigenvalue $(\lambda 1)$ of COA and between and within COA analysis for each sampling dates : Site of Bréhémont (1996)

\begin{tabular}{|l|c|c|c|c|}
\hline Variables & Octobre - février & Mars - avril & Mai - juin & Juillet - sept. \\
\hline AFC simple & $\boldsymbol{\lambda} \mathbf{1}=\mathbf{0 , 3 8}$ & $\boldsymbol{\lambda} \mathbf{1}=\mathbf{0 , 2 8}$ & $\boldsymbol{\lambda} \mathbf{1}=\mathbf{0 , 3 6}$ & $\boldsymbol{\lambda} \mathbf{1}=\mathbf{0 , 4 4}$ \\
\hline AFC inter substrat/vitesse & 0,23 & 0,20 & 0,21 & 0,33 \\
\hline AFC inter vitesse & 0,23 & 0,18 & 0,20 & 0,30 \\
\hline AFC inter substrat & 0,18 & 0,14 & 0,14 & 0,21 \\
\hline AFC inter profondeur & 0,04 & 0,08 & 0,05 & 0,07 \\
\hline
\end{tabular}

Tableau VI. - Importance relative de la première valeur propre $(\lambda 1)$ de chaque variable analysée par AFC inter-classe (en \% par rapport à la première valeur propre de l'AFC globale) pour les différentes périodes de l'année et pour toute l'année 1996 sur le site de Bréhémont.

Table VI. - Eigenvalue of between COA analysis (\% / global COA analysis).

\begin{tabular}{|l|c|c|c|c|c|}
\hline Variables & $\begin{array}{c}\text { Octobre - } \\
\text { février }\end{array}$ & Mars - avril & Mai - juin & $\begin{array}{c}\text { Juillet - } \\
\text { sept. }\end{array}$ & $\begin{array}{c}\text { Année } \\
\mathbf{1 9 9 6}\end{array}$ \\
\hline AFC inter substrat/vitesse & $60,5 \%$ & $71,4 \%$ & $58,3 \%$ & $75 \%$ & $\mathbf{5 8 , 3} \%$ \\
\hline AFC inter vitesse & $60,5 \%$ & $64,3 \%$ & $55,6 \%$ & $68,2 \%$ & $\mathbf{5 2 , 8} \%$ \\
\hline AFC inter substrat & $47,4 \%$ & $50 \%$ & $38,9 \%$ & $47,7 \%$ & $\mathbf{3 8 , 9} \%$ \\
\hline AFC inter profondeur & $10,5 \%$ & $28,6 \%$ & $13,3 \%$ & $15,9 \%$ & $\mathbf{1 3 , 9} \%$ \\
\hline
\end{tabular}

Les graphes factoriels des analyses inter-substrat-vitesse (Fig. 8) montrent toutefois de fortes variations des structures de l'habitat au cours de l'année. Très perturbés d'octobre à février et encore plus en mars-avril lors des fortes crues printanières (Fig. 3), ces dernières ne se structurent vraiment que du mois de mai au mois de septembre.

\subsection{Comparaison La Charité-sur- Loire/Bréhémont}

II semble intéressant de vérifier si les résultats obtenus sont généralisables à l'ensemble du cours de la Loire. Aussi, la même analyse a été conduite sur le site de La Charité-sur-
Loire. La comparaison présentée ici donne les résultats globaux trouvés sur les deux sites à partir de l'ensemble des échantillons prélevés. Les analyses des prélèvements réalisés aux mêmes dates et en ne retenant que les espèces communes aux deux sites corroborent ces résultats (Bacchi, 2000).

Les résultats obtenus sur le site de La Charité-sur-Loire sont représentés dans le tableau VII.

L'analyse des facteurs de microdistribution à l'échelle stationnelle met en évidence que la micro-distribution des taxons sur le site de La Charité-sur-Loire est influencée de préférence par la nature du substrat plutôt que par la vitesse de courant. 


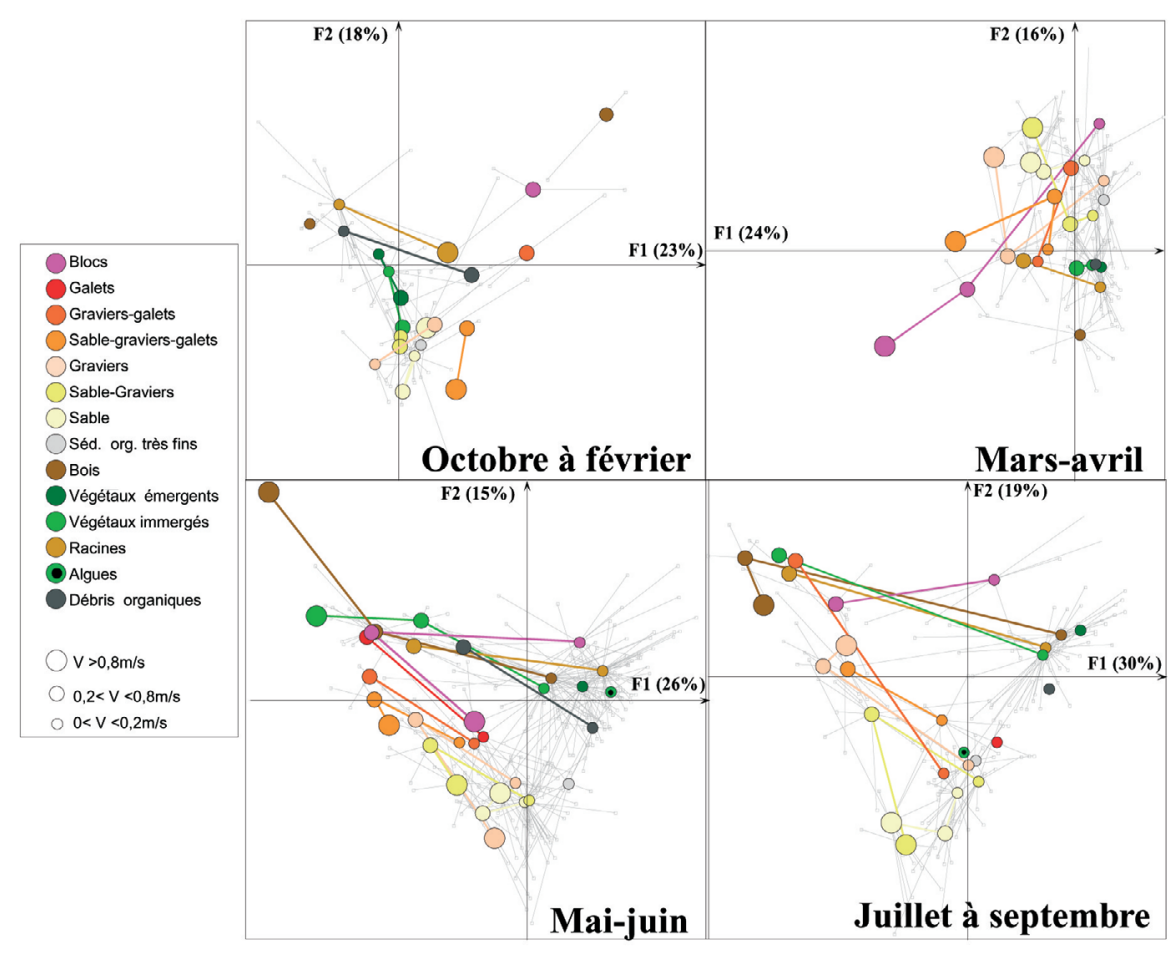

Fig. 8. - Premier plan factoriel des AFC inter-classe substrat-vitesse de courant réalisées à partir des relevés faunistiques sur le site de Bréhémont. Analyse de la variabilité temporelle.

Fig. 8. - F1-F2 factorial plane of between COA analysis (substrat and current velocity). Site of Bréhémont.

Fig. 9. - Premier plan factoriel des AFC inter-classe du tableau (200 lignes prélèvements, 71 colonnes taxons) des prélèvements réalisés sur le site de La Charité-sur-Loire en 1996.

VE : Histogramme des valeurs propres AFC inter-substrat.

VF : Histogramme des valeurs propres de l'AFC inter-substrat-vitesse.

Ea : Position des centres d'inertie des substrats dans l' AFC inter-substrat.

Eb : Plan factoriel des taxons dans l'AFC inter-substrat.

Fa : Position des centres d'inertie des substrats-vitesses dans l'AFC inter-substrat-vitesse.

$\mathrm{Fb}$ : Plan factoriel des taxons dans l'AFC inter-substrat-vitesse.

Fig. 9. - F1-F2 factorial plane of between COA analysis Projection of variables and taxa on the first factorial plane. Site of La Charité-sur-Loire (1996).

VE : Eigenvalue diagram of between substrats COA analysis.

VF : Eigenvalue diagram of between substrat/current velocity COA analysis.

Ea : Results of the between-substrats COA analysis. Factorial plane (1-2) of samples separated by substrats.

Eb : Results of the between-substrats COA analysis. Factorial plane (1-2) of fauna.

Fa : Results of the between substrat/current velocity COA analysis. Factorial plane (1-2) of samples separated by substrat/current velocity.

$\mathbf{F b}$ : Results of the between substrat/current velocity COA analysis. Factorial plane (1-2) of fauna. 


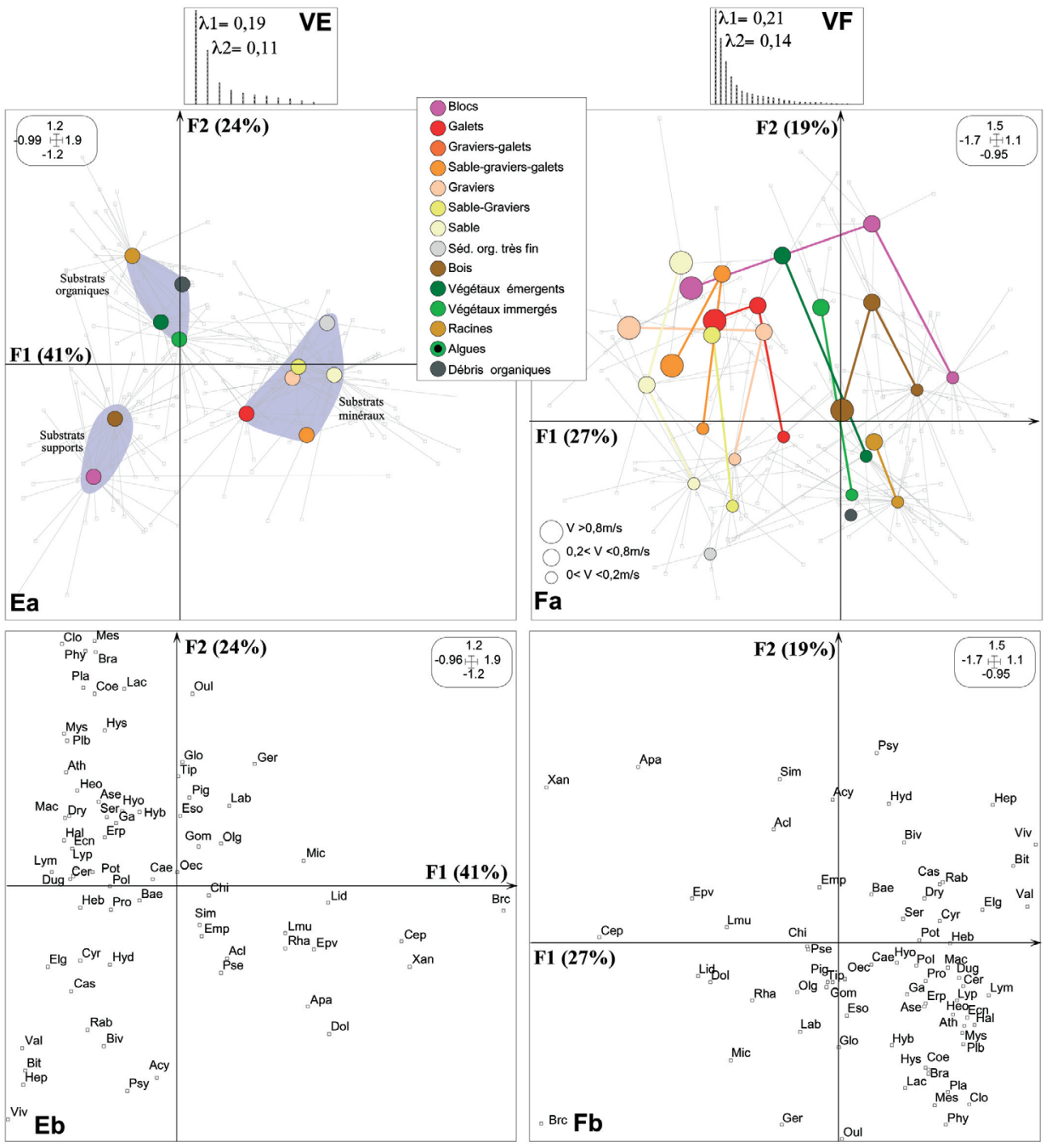

Fig. 9 
Tableau VII. - Valeur de la première valeur propre $(\lambda 1)$ et importance relative de chaque variable analysée par AFC inter-classe (en \% par rapport à la première valeur propre de l'AFC globale) pour toute l'année 1996 sur les sites de Bréhémont et de La Charité-sur-Loire.

Table VII. - Compared eigenvalue of COA and between COA analysis. Site of Bréhémont and site of La Charité-sur-Loire.

\begin{tabular}{|l|c|c|c|c|}
\hline Variables & \multicolumn{2}{|c|}{ La Charité-sur-Loire } & \multicolumn{2}{c|}{ Bréhémont } \\
\hline AFC simple & \multicolumn{2}{|c|}{$\boldsymbol{\lambda 1}=\mathbf{0 , 3 1}$} & \multicolumn{2}{c|}{$\boldsymbol{\lambda 1}=\mathbf{0 , 3 6}$} \\
\hline AFC inter-substrat/vitesse & $\mathbf{0 , 2 1}$ & $\mathbf{6 7 , 7} \%$ & $\mathbf{0 , 2 1}$ & $\mathbf{5 8 , 3} \%$ \\
\hline AFC inter-vitesse & 0,18 & $58,1 \%$ & $\mathbf{0 , 1 9}$ & $\mathbf{5 2 , 8} \%$ \\
\hline AFC inter-substrat & $\mathbf{0 , 2 0}$ & $\mathbf{6 4 , 5} \%$ & 0,14 & $38,9 \%$ \\
\hline AFC inter-rive/chenal & 0,04 & $12,9 \%$ & 0,05 & $13,9 \%$ \\
\hline
\end{tabular}

Cette différence entre les deux sites analysés s'exprime, en premier lieu, par des différences structurelles entre les facteurs de micro-distribution et notamment pour le substrat et le couple substrat-vitesse. Ainsi, si globalement la hiérarchie de la richesse taxinomique des différents substrats peut être considérée comme équivalente sur les deux sites, l'image obtenue par AFC interclasses n'est pas semblable. Si l'on retrouve, comme sur le site de Bréhémont, une opposition entre substrats organiques et minéraux, on remarque aussi (Fig. 9, Ea et Eb) :

- des substrats «support», les bois et les blocs, qui hébergent un cortège faunistique particulier,

- la moindre différenciation du cortège faunistique en fonction d'un gradient hétérométrique pour les substrats minéraux sur le site de La Charité-sur-Loire.

La structure obtenue pour le couple substrat vitesse est aussi très différente de celle qui a été observée sur Bréhémont puisque l'on note (Fig. 9, Fa et Fb) une plus grande dis- persion dans le plan factoriel des substrats échantillonnés dans des vitesses de courant élevées (classe de vitesse 3 supérieure à $0,8 \mathrm{~m} / \mathrm{s}$ ). La part relative prise dans la structuration des peuplements par les différentes variables (Tableau VII) montre très clairement la part plus importante que prend le couple substrat-vitesse, et notamment le substrat, dans la structuration des peuplements sur le site de La Charité-sur-Loire.

Ces différences structurelles confirment la nécessité pour un certain nombre de taxons du site de Bréhémont de préférer des conditions rhéophiles plus favorables à leur survie dans un environnement perturbé. Cette condition n'apparaît pas nécessaire sur le site de La Charité-sur-Loire où la qualité de l'eau est bien meilleure : la nature du substrat devient alors prépondérante dans la distribution des taxons à l'échelle stationnelle.

Ce travail de comparaison souligne aussi très clairement l'intérêt d'analyser les peuplements à différentes échelles pour déterminer, no- 
tamment sur le site de Bréhémont, comment d'autres facteurs peuvent intervenir dans la structuration des peuplements. Dans ce cadre, l'analyse conjointe des facteurs de macro et de micro-distribution apparaît comme une nécessité (Bacchi, in prép.).

\section{CONCLUSION}

Les différentes études des peuplements ligériens menées dans le cadre du Plan Loire Grandeur Nature, montrent que malgré un fonctionnement complexe lié à des particularités morphologiques, géologiques, climatiques et à une anthropisation affectant notablement les caractéristiques physiques et physico-chimiques de cet hydrosystème, celui-ci possède encore un fort potentiel biologique qui justifie pleinement l'intérêt que l'on doit y porter.

L'analyse des facteurs de microdistribution de la macrofaune benthique à l'échelle des sites présentés ici, montre qu'en dehors de l'influence respective des facteurs de macro-distribution (lit vif et annexes hydrauliques) présentée par de nombreux auteurs (Bournaud et al., 1983 ; Bournaud et al., 1987 ; Franquet et al., 1995 ; Bacchi, 2000...) et souvent sous l'influence de facteurs externes fortement perturbateurs comme les crues (Gaschignard, 1984 ; Cellot et al., 1986 ; Resh et al., 1988...), la structuration des peuplements dépend aussi de facteurs de micro-distribution.
Les paramètres de micro distribution les plus structurants dont l'importance est démontrée depuis fort longtemps, (Thienemann, 1912 ; Shelford, 1918; Behning, 1928; Muttkowsky, 1929 ; Gerbacher, 1937 ; Illies \& Botosaneanu, 1963; Verneaux, $1973, \ldots)$, sont analysés ici à l'échelle stationnelle avec une attention toute particulière pour le rôle joué, au cours de l'année, par le substrat ou la vitesse de courant. Outre une partie descriptive, ce travail a aussi permis, par l'utilisation des Analyses Factorielles des Correspondances inter et intra classes, de hiérarchiser entre eux ces différents paramètres.

Ainsi, l'étude de la variété taxinomique par substrat montre de très fortes similarités entre les deux sites ligériens de Bréhémont et de La Charité-sur-Loire. Celle-ci est maximum pour les substrats organiques (avec par ordre d'importance les racines, les végétaux émergents, les végétaux immergés, les bois, les débris organiques) et moins forte pour les substrats minéraux (blocs, galets, galets-graviers, sédiments fins très organiques, sables-graviers-galets, graviers, sables-graviers et les sables).

Sur chaque site, les principaux facteurs connus de micro-distribution de la faune benthique ont été analysés.

Sur le site de Bréhémont, pour l'année 1996, on a pu démontrer que le couple substrat-vitesse apparaît comme le principal facteur de microdistribution des invertébrés aquatiques. Toutefois, la présence des taxons est davantage liée à la vitesse 
de courant qu'à la nature du substrat. La recherche systématique pour certains taxons de conditions rhéophiles marquées, semblerait prouver l'influence de facteurs limitants qui imposeraient un habitat réduit :

- L'analyse de la structure substrat montre deux pôles distincts, l'un organique et l'autre minéral. Si les substrats organiques abritent les mêmes taxons, il n'en va pas de même pour les substrats minéraux dont le peuplement change en fonction de la granularité et plus particulièrement en fonction de la fraction granulométrique la plus grossière.

- L'analyse temporelle montre que la structure faunistique évolue au cours de l'année. Bien que les structures temporelles d'organisation de la faune soient très différentes d'une période à une autre, la hiérarchie entre les différents facteurs est conservée.

Sur le site de La Charité-sur-Loire, contrairement à ce qui a pu être observé sur le site de Bréhémont, la micro-distribution des taxons est influencée préférentiellement par la nature du substrat et non par la vitesse de courant. Cette différence entre les deux sites s'exprime aussi par des différences entre les structures de micro-distribution, notamment en ce qui concerne le substrat et le couple substrat-vitesse.

L'étude conjointe des facteurs de micro-distribution sur les deux sites montre que si le couple substrat-vitesse apparaît toujours, en dehors des périodes de crues, comme le facteur qui explique le mieux la micro-distribu- tion des taxons, par contre, suivant les sites analysés, la hiérarchie entre ces deux facteurs peut être inversée sous l'influence de l'action d'un facteur s'exerçant à une dimension plus grande: I'hypothèse de la dégradation de la qualité de l'eau, sur le secteur de Bréhémont, qui impose à certaines espèces des conditions rhéophiles plus marquées pour pouvoir survivre dans un contecte hypertrophe très marqué (recherche des habitats oxygénés dans les secteurs rhéophiles de la rivière) est avancée. Toutefois, il n'est pour l'instant pas envisageable de caractériser le ou les facteurs mésologiques principaux $(\mathrm{pH}$, oxygène, température, MES, débit,...) intervenant dans les modifications structurales observées. En effet, le déterminisme des facteurs influents en écologie se heurte, notamment sur des écosystèmes d'importance soumis à de très fortes variabilités spatiales et temporelles, à la relativité de l'influence respective des paramètres structurants, puisque ceux-ci sont très souvent fortement corrélés.

Cette différence dans l'occupation des niches écologiques par des peuplements similaires, dans des conditions mésologiques générales semblables (typologie équivalente) a pu être confirmée sur une partie du peuplement (Plécoptères, Ephéméroptères et Trichoptères) avec un niveau de détermination spécifique et en ne retenant que les espèces communes aux deux sites pendant la même période d'échantillonnage (Bacchi, 2000). Elle pose la question de la seule utilisation des traits biologi- 
ques pour l'évaluation de la qualité des hydrosystèmes (Bournaud et al., 1992 ; Chevenet et al., 1994 ; Doledec \& Chessel, 1994 ; Usseglio-Polatera, 1994). En effet, il apparaît ici que les impacts anthropiques, plus ou moins importants d'amont en aval, ne conduisent pas nécessairement à un remplacement d'espèces et donc à une modification de la structure globale des traits biologiques de l'ensemble du peuplement, mais peut provoquer des changements imposés d'habitats sous l'effet des contraintes du milieu et donc une répartition des peuplements en fonction de paramètres de micro-distribution différents (vitesse de courant plutôt que nature du substrat par exemple). La seule analyse des traits biologiques pourrait alors s'avérer insuffisante pour caractériser des perturbations de faible ampleur ou s'appliquant à des échelles spatiales et/ou temporelles réduites.

Une analyse des traits biologiques sur ces deux stations sera conduite prochainement pour vérifier cette hypothèse.

Outre un intérêt scientifique certain, l'objectif à long terme des recherches entreprises sur la macrofaune benthique du système potamique ligérien, dont l'objet est de déterminer «l'essentiel », commun ou non, de l'organisation spatiale des communautés à l'échelle d'un site ou d'une station, est aussi susceptible d'intéresser les gestionnaires qui ont en charge la restauration et l'entretien de tels hydrosystèmes :

- d'une part, parce que la compréhension globale des interactions entre facteurs mésologiques et peuplements présents est un préalable indispensable pour déterminer la réactivité possible de l'hydrosystème et, dans un deuxième temps, pour mesurer les effets d'une perturbation,

- d'autre part, parce que ce travail pourrait servir de base pour concevoir une méthode d'analyse des hydrosystèmes potamiques, soit par la mise au point d'un protocole d'échantillonnage stratifié, soit par la caractérisation d'une typologie structurelle et fonctionnelle (analyse conjointe des facteurs de macro et de micro distribution) utile dans des études d'impacts qui relèveraient de la biocénotique approfondie (Bacchi, in prép.).

\section{BIBLIOGRAPHIE}

Agence de l'Eau Loire Bretagne, 1994 Cartes de qualité des cours d'eau du bassin Loire Bretagne, connues au 1er janvier 1994 - 3 cartes.

Andersen R.V. \& David M.D., 1986 - Prédictive quality of macroinvertebrate - habitat associations in lower navigation pools of the Mississipi River. Hydrobiologia 136, 101-112.

Bacchi M., 2000 - Structure et dynamique des peuplements macrobenthiques en Loire. Impact des facteurs hydrologiques et sédimentaires. Mèm. Thèse Doctorat. Université de Tours, $252 \mathrm{pp}$.

Becker G., 1994 - Food preference by five trichopteran scrapers. Hydrobiologia 273, 171-178.

Behning A., 1928 - Das leben der Wolga. Zugleich eine Einführung in die FluBBiologie. Die Binnengewässer, 5 . Stuttgart. 
Beisel J.N., 1996 - Microrépartition des invertébrés benthiques en eau courante : caractéristiques des microhabitats et organisation de leurs peuplements. Thèse de $3^{\text {ème }}$ cycle. Université de Metz, 242 pp.

Bournaud M. \& Cogerino L., 1986 - Les microhabitats des rives d'un grand cours d'eau: Approche faunistique. Annls. Limnol., 22(3), 285-294.

Bournaud M., Maucet D. \& Chavanon G., 1983 - Structure des peuplements de macroinvertébrés benthiques en place et en dérive. Acta Oecologica. Oecol. Gener. 4(1), 27-42.

Bournaud M., Tachet H. \& Roux A.L., 1987 - The effect of seasonal and hydrological influences on the macroinvertebrates of the Rhône River (France). II Ecological aspects. Arch. Hydrobiol./Suppl.76 (1/2), 25-51.

Bournaud M., Richoux P. \& Usseglio-Polatera P., 1992 - An approach to the synthsis of qualitative ecological information from aquatic coleoptera communities. Regulated Rivers 7: 165180.

Cellot B. \& Bournaud M., 1986 - Modifications faunistiques engendrées par une faible variation de débit dans une grande rivière. Hydrobiologia 135, 223-232.

Chessel D., Thioulouse J., Dolédec S. \& Olivier J.M., 1993 - Software. Multivaraite Analysis and graphical display for environmental data. Version 4. HyperCard stacks and program library for the analysis of environmental data. U.R.A. C.N.R.S.1451 «Ecologie des Eaux Douces et des Grands Fleuves ». Université Claude Bernard Lyon 1, France.

Chevenet F., Dolédec S. \& Chessel D., 1994 - A fuzzy coding approach for the analysis of long term ecological data. Freshwat. Biol., 31 : 295-309.

Chovet M. \& Lécureuil J.Y., 1994 - Répartition des Gammaridae épigés (Crustacés, Amphipodes) dans la
Loire et les rivières de la Région Centre (France). Annls. Limnol. 30, 1124.

Chovet M., Fontaine J. \& Lécureuil J.Y., 1984 - Le genre Ephemerella Walsh dans le bassin de la Loire: Présence de deux espèces nouvelles pour la France : E. notata Eaton et E. mesoleuca Brauer (Epheméroptères ; Ephemerellidae) In V. Landa (ed.) Proceeding of the Fourth International Conférence on the Ephemeroptera, 117-126.

Cogerino L., 1989 - Les rives aquatiques de grands cours d'eau : Caracctérisation mésologique et faunistique. Thèse $3^{\text {ème }}$ cycle. Univisersité Claude-Bernard Lyon I. 369 pp.

Cornier T., 1998 - Essai de typologie écologique des communautés végétales du lit de la Loire. Univ. De Tours, Diren Centre, Orléans, 47 pp.

Cummins K.W. \& Lauff G.H., 1969 - The influence of substrate particle size on the microdistribution of stream macrobenthos. Hydrobiologia, 34 (2), 145181.

Dambre J.L. \& Malaval P., 1993 - Evaluation des conditions de poursuite de la politique de limitation des extractions de matériaux dans le lit de la Loire du Bec d'Allier à Nantes. Paris, mission d'inspection des services de la navigation, mission d'inspection spécialisée de l'Equipement, 56p.

Degiorgi F., 1994 - Etude de l'organisation spatiale de l'ichtyofaune lacustre. Prospection multisaisonnière de 6 plans d'eau de l'est de la France à l'aide de filets verticaux. Mèm. Thèse $3^{\text {ème }}$ cycle, Univ. Fr. Comté, 207p.

Doledec S. \& Chessel D., 1989 - Rythmes saisonniers et composante stationnelles en milieu aquatique. II. Prise en compte et élimination d'effets dans un tableau faunistique. Acta Oecol., Ecol. Gener., 10, 3, 207-232.

Doledec S. \& Chessel D., 1994 - Co-inertia analysis ; an alternative method for 
studying species environment relationships. Freshwat. Biol., 31 : 277293.

Favet C., 1981 - Communautés ripicoles de la basse Durance. Thèse $3^{\text {ème }}$ cycle, Aix-Marseille 3, $371 \mathrm{pp}$.

Ferry C. \& Frochot B., 1970 - L'avifaune nidificatrice d'une forêt de Chênes pédonculés en Bourgogne: étude de deux successions écologiques. $\mathrm{La}$ terre et la vie 24, 153-250.

Franquet E., Cellot B., Pont D. \& Bournaud M., 1995 - Environmental and macroinvertébrate dynamics in the Lower Rhone River and a lateral dike field : a study matching two functioning descriptors. Hydrobiologia 308, 207217.

Garcia X.F., 1994 - Premier inventaire faunistique des Chironomidés (Diptera) du potamal de la Loire et du Cher Région de Tours - Tronçon d'ordre six - Quelques aspects écologiques du peuplement. Mémoire de DEA, Université Paul Sabatier, Toulouse, 35 pp. + annexes.

Garcia X.F. \& Laville H., 1999a - First inventory and faunistic originality of the Chironomidae (Diptera) from a 6th order section of the river Loire (France). Archiv. hydrobiol.

Garcia X.F. \& Laville H., 1999b - Spatiotemporal distribution of the chironomid populations in the lower part of a large river : the middle Loire (France). Verh. Internat. Verein. Limnol.

Gaschignard O., 1984 - Impact d'une crue sur les macroinvertébrés benthiques d'un bras du Rhône. Verh. Internat. Verein. Limnol. 22, 1997-2001.

Gerbacher W.M., 1937 - The development of stream bottom communities in Illinois. Ecology 18, 359-390.

Guidicelli, 1968 ? ?

Guinand B., Ivol J.M. \& Tachet H., 1998 - Longitudinal distribution of Trichoptera in the Loire River (France) : simple ordination methods and community structure. Hydrobiologia 317, 231-245.
Grosbois C., 1998 - Géochimie des eaux de la Loire : contributions naturelles et anthropiques. Quantification de l'érosion. Mèm. Thèse de Doctorat. Université de Tours : 232 pp.

Hynes H.B.N., 1970 - The ecology of running waters. Liverpool University Press, Liverpool, 555 pp.

Illies J. \& Botosaneanu L., 1963 - Problèmes et méthodes de la classification et de la zonation écologique des eaux courantes, considérées surtout du point de vue faunistique. Internationale vereinigung für theoretische und angewandte limnologie. Komitee für limnologische methoden. №12.

Iversen T.M., 1974 - Ingestion and growth in Séricostoma personatum (Trichoptera in relation to the nitrogen content of ingested leaves. Oïkos 25 (3), 278-282.

Ivol J.M., Guinand B., Richoux P. \& Tachet H., 1997 - Longitudinal changes in Trichoptera and coleoptera assemblages and environmental conditions in the Loire River (France). Arch. Hydrobiol. 138 (4), 525-527.

Jenkins R.A., Wade K.R. \& Pugh E., 1984 - Macroinvertebrate-habitat relationship in the river Teifi catchment and the significance to conservation. Freshwat. Biol., 14 (1), 23-42.

Lapchin L., 1977 - Microrépartition des invertébrés benthiques dans deux ruisseaux à salmonidés de Bretagne. Ann. Hydrobiol. 8 (3), 319-332.

Lécureuil J.Y., Chovet M., Bournaud M. \& Tachet H., 1983 - Description, répartition et cycle biologique de la larve d'Hydropsyche bulgaromanorum MALICKY, 1977 (Trichoptera Hydropsychidae) dans la basse Loire. Annls Limnol. 19, 17-24.

Lécureuil J.Y., Fontaine J. \& Chovet M., 1984 - Nouvelles données sur l'écologie de Raptobaetopus tenellus (ALBARDA), un Baetidae présent en grand nombre dans le bassin de la Loire (France) - In Landa V., Soldan 
T., Tonner M. (Eds.) : Proc Ivth intern. Confer. Ephemeroptera, 4.-10. September 1983, Bechyne (Czechoslovakia), Czeschoslovak Academy of Sciences. 253-261.

Muttkowsky R.A., 1929 - The ecology of trout streams in Yellowstone National Park. Roosevelt Wild Life. Ann 2, 155240.

Perrin J.F., 1978 - Signification écologique des peuplements benthiques du Haut-Rhône Français. Thèse de $3^{\text {ème }}$ cycle, Lyon I. 171 pp.

Prodon R., 1976 - Le substrat, facteur écologique et éthologique de la vie aquatique : observations et expériences sur les larves de Micropterna testacea et Cordulegaster annulatus. Thèse de $3^{\text {ème }}$ cycle. Lyon I, 221 pp.

Resh V.H., Brown A.V., Covich A.P., Gutz M.E., Hiram W.L., Minshall G.W., Reice S.R., Sheldon A.L., Wallace J.B. \& Wissmar R.C., 1988 - The role of disturbance in stream ecology. J.N. Amer. Benthol. Soc. 7, 433-455.

Shelford V.E., 1918 - Conditions of existence. In Ward \& Wipple, Freschw. Biol., 21-60.

Thienemann A., 1912 - Der Bergbach des Sauerlandes. Faunistisch-biologische
Untersuchungen. Internat. Rev. Biol. Suppl. 4, 1-125.

Usseglio-Polatera P., 1994 - Theoretical habitat templets species traits, and species richness: aquatic insects in the Upper Rhône River and its floodplain. Freshwat. Biol., 31 : 417-437.

Verneaux J., 1973 - Cours d'eau de Franche-Comté (massif du Jura). Recherches écologiques sur le réseau hydrographique du Doubs; essai de biotypologie. Thèse Faculté des Sciences de Besançon, 257 pp.

Verneaux J., 1984 - Méthodes biologiques et problèmes de la détermination des qualités des eaux courantes. Bull. Ecol. 15, 1, 47-55.

Verneaux J., 1994 - Variables biologiques : des indicateurs de l'état de santé des écosystèmes aquatiques. Séminaire National. GIP hydrosystèmes.

Verneaux V., 1996 - Structure, dynamique spatiale et temporelle du peuplement chironomidien du lac de l'Abbaye (massif du Jura) et approche typologique. Relation entre la typologie larvaire et la structuration des peuplements émergents. Mèm. Thèse Doctorat, Univ. Fr. Comté, 187p.

\section{ANNEXE I}

Annexe I. - Codes des Familles (MAJUSCULES) ou des genres (italique) utilisés pour la représentation graphique des taxons dans les analyses inter-classes. Les taxons sont classés par ordre alphabétique des codes utilisés.

Annexe I. - FAMILLY and genera number code used in within and between COA analysis

\begin{tabular}{|ll|ll|ll|ll|}
\hline Code & FAMILLE/genre & Code & FAMILLE/genre & Code & FAMILLE/genre & Code & FAMILLE/genre \\
\hline Aci & Acilius & Dug & Dugesia & Hyo & Hydroptila & Ple & Plea \\
\hline Acl & Acentrella & Dup & Dupophilus & Hyp & Hydrophilus & Plm & Platambus \\
\hline Acy & Ancylus & Ech & Echinogammarus & Hyr & Hydroporus & Pof & Polycentropus \\
\hline Ago & Agabus & Ecn & Ecnomus & Hys & Hydrometra & Pol & Potamanthus \\
\hline
\end{tabular}




\begin{tabular}{|c|c|c|c|c|c|c|c|}
\hline Code & FAMILLE/genre & Code & FAMILLE/genre & Code & FAMILLE/genre & Code & FAMILLE/genre \\
\hline Ana & Anacaena & Elg & Electrogena & $\mathrm{Ili}$ & Ilybius & Pot & Potamophilus \\
\hline Ano & Anodonta & Elm & Elmis & Ily & Ilyocorus & Pro & Procloeon \\
\hline Ant & ANTHOMIIDAE & Emp & EMPIDIDAE & Lab & Laccobius & Psc & PSYCHODIDAE \\
\hline Apa & Aphelocheirus & Eno & Enochrus & Lac & Laccophilus & Pse & $\begin{array}{l}\text { Pseudocentrop- } \\
\text { tilum }\end{array}$ \\
\hline Ase & Asellus & Eph & EPHYDRIDAE & Les & LESTIDAE & Psy & Psychomyia \\
\hline Atd & Atyaephyra & Epv & Ephoron & Leu & Leuctra & Rab & Raptobaetopus \\
\hline Ath & ATHERICIDAE & Ero & Erotesis & Lib & LIBELLULIDAE & Rha & RHAGIONIDAE \\
\hline Atp & Athripsodes & Erp & Erpobdella & Lid & LIMONIIDAE & Rio & Riolus \\
\hline Bae & Baetis & Eso & Esolus & Lim & Limnephilus & Sal & SALDIDAE \\
\hline Ber & Berosus & Fer & FERRISSIIDAE & Lmu & Limnius & Sca & Scarodytes \\
\hline Bit & BITHYNIIDAE & Gam & Gammarus & Lsp & stade 2 & Ser & Serratella \\
\hline Biv & BIVALVES & Ger & Gerris & Lym & LYMNAEIDAE & Set & Setodes \\
\hline Bra & Brachycentrus & Glo & Glossiphonia & Lyp & Lype & Sia & Sialis \\
\hline $\mathrm{Brc}$ & Brachycercus & Gly & Glyphotaelius & Mac & Macronychus & Sim & SIMULIIDAE \\
\hline Bry & Brychius & Gom & GOMPHIDAE & Mel & Maladema & Sph & Siphlonurus \\
\hline Cae & Caenis & Hae & HYDRAENIDAE & Mes & MESOVELIIDAE & Ste & Stenelmis \\
\hline Cas & Calopteryx & $\mathrm{Hal}$ & Halesus & Mic & Micronecta & Syr & SYRPHIDAE \\
\hline Cep & CERATOPOGONIDAE & $\mathrm{Hal}$ & Haliplus & Mys & Mystacides & Tab & TABANIDAE \\
\hline Cer & Ceraclea & $\mathrm{Hbp}$ & habrophlebia & Neb & Neureclipsis & Tae & Taeniopteryx \\
\hline Cet & Centroptilum & Hch & HYDROCHIDAE & $\mathrm{Nec}$ & Nepa & Tip & TIPULIDAE \\
\hline Cha & Chaetarthria & Hdd & HYDRIDAE & Nor & Normandia & Uni & Unio pictorum \\
\hline Che & Cheumatopsyche & $\mathrm{Heb}$ & Helobdella & Not & Notonecta & Val & VALVATIDAE \\
\hline Chi & CHIRONOMIDAE & Hei & Helichus & Oec & Oecetis & Viv & VIVIPARIDAE \\
\hline Chp & Chloroperla & $\mathrm{Hel}$ & Helochares & Olg & OLIGOCHETES & Yol & Yola \\
\hline $\mathrm{Clb}$ & Colymbetes & Heo & Helophorus & Oli & Oligoneuriella & & \\
\hline Clo & Cloeon & Hep & Heptagenia & Olp & Oligoplectrum & & \\
\hline Coe & COENAGRIONIDAE & Hir & HIRUDIDAE & Orl & Orconectes & & \\
\hline Col & Coelambus & $\mathrm{Hol}$ & Holocentropus & Oul & Oulimnius & & \\
\hline Cor & Corixinae & $\mathrm{Hpd}$ & Hyphidrus & Pel & Peltodites & & \\
\hline Cul & CULICIDAE & Hya & HYDRACARIENS & Phy & PHYSIDAE & & \\
\hline Cyr & Cyrnus & Hyb & HYDROBIIDAE & $\mathrm{Pig}$ & Piscicola & & \\
\hline Den & Dendrocoelum & Hyc & Hydaticus & Pis & Pisidium & & \\
\hline Dol & DOLICHOPODIDAE & Hyd & Hydropsyche & Pla & Platycnemis & & \\
\hline Dry & Dryops & Hyi & Hydrobius & $\mathrm{Plb}$ & PLANORBIDAE & & \\
\hline
\end{tabular}


